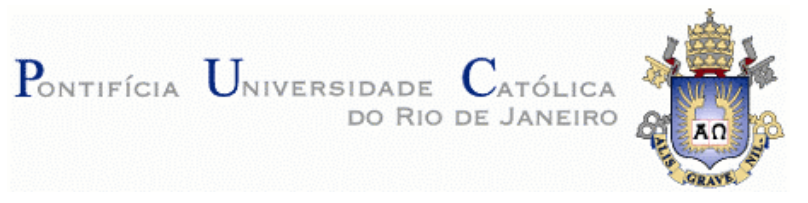

Elizete Gonçalves Lopes Rangel

\title{
Normalização em metrologia no Comando da Aeronáutica: diagnóstico e considerações sobre um modelo integrado.
}

\section{Dissertação de Mestrado}

Dissertação apresentada como requisito parcial para obtenção do título de Mestre pelo Programa de PósGraduação em Metrologia da PUC-Rio. Área de Concentração: Metrologia para Qualidade e Inovação.

Professores Orientadores:

Maurício Nogueira Frota, Ph.D. PósMQI/PUC-Rio

Álvaro José Damião, Dr. CTA/IEAv, São José dos Campos, SP

Rio de Janeiro maio de 2005 
Elizete Gonçalves Lopes Rangel

Normalização em metrologia no Comando da Aeronáutica: diagnóstico e considerações sobre um modelo integrado.

Dissertação apresentada como requisito parcial para obtenção do grau de Mestre pelo Programa de Pós-Graduação em Metrologia do Centro Técnico Científico da PUC-Rio. Aprovada pela Comissão Examinadora e homologada pela Coordenação Setorial de Pós-Graduação, formalizado pelas respectivas assinaturas:

Comissão Examinadora:

Prof. Dr. Maurício Nogueira Frota Orientador Programa de Pós-Graduação em Metrologia (PósMQI/PUC-Rio) Pontifícia Universidade Católica do Rio de Janeiro

Prof. Dr. Álvaro José Damião

Co-orientador Instituto de Estudos Avançados do Centro Técnico Aeroespacial (CTA/IEAv), São José dos Campos, SP

Prof. Dr. Paula M. P. Gouvêa Programa de Pós-Graduação em Metrologia (PósMQI/PUC-Rio) Pontifícia Universidade Católica do Rio de Janeiro

Prof. Dr. Roberto Peixoto Departamento de Engenharia Industrial (PUC-Rio) Pontifícia Universidade Católica do Rio de Janeiro

M.Sc Luis Guilherme Gigo Instituto de Fomento e Coordenação Industrial do Centro Técnico Aeroespacial (CTA/IFI), São José dos Campos, SP

Coordenação Setorial de Pós-Graduação:

Prof. José Eugênio Leal Coordenador Setorial de Pós-Graduação do Centro Técnico Científico (PUC-Rio) Rio de Janeiro, 05 de maio de 2005. 
Todos os direitos reservados. É proibida a reprodução total ou parcial do trabalho sem autorização da universidade, da autora e do Professor orientador.

\section{Elizete Gonçalves Lopes Rangel}

Graduada em Engenharia Elétrica-eletrônica pela Faculdade de Ciências Exatas da Fundação Valeparaibana de Ensino de São José dos Campos, em 1990. Engenheira do Instituto de Atividades Espaciais (IAE/CTA), de 1991 a 1995. Engenheira do Instituto de Pesquisas Espaciais (INPE) de 2001 a junho de 2002. Engenheira do Instituto de Fomento e Coordenação Industrial (IFI/CTA), de julho de 2002 a abril de 2005. Integrada ao quadro permanente do Instituto de Pesquisas Espaciais (INPE) a partir de maio de 2005.

Ficha Catalográfica

Rangel, Elizete Gonçalves Lopes

Normalização em metrologia no Comando da Aeronáutica: diagnóstico e considerações sobre um modelo integrado / Elizete Gonçalves Lopes Rangel; orientadores: Maurício Nogueira Frota; Álvaro José Damião. - Rio de Janeiro: PUC-Rio, Departamento de Metrologia para Qualidade Industrial, 2005.

196 f. : il. ; $30 \mathrm{~cm}$

Dissertação (mestrado) - Pontifícia Universidade Católica do Rio de Janeiro, Departamento de Metrologia para Qualidade Industria.

Inclui referências bibliográficas

1. Metrologia - Teses. 2. Normalização em metrologia. 3. Sistema de metrologia aeroespacial. 4. Elaboração de normas. 5. Pesquisa de demanda por normas em metrologia no Comando da Aeronáutica. I. Frota, Maurício Nogueira. II. Damião, Álvaro José. III. Pontifícia Universidade Católica do Rio de Janeiro. Departamento de Metrologia para Qualidade. IV. Título. 


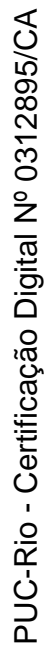

Dedico este trabalho a meu esposo Marcelo e aos meus queridos filhos Victor e Luíza. 


\section{Agradecimentos}

Aos meus pais, pelo carinho e apoio.

Ao Professor Maurício Frota, pela presente orientação que contribuiu não só para o desenvolvimento deste trabalho, como também para meu aperfeiçoamento profissional.

Ao Dr. Álvaro Damião (CTA/IEAv), pela co-orientação e apoio colaborando para a realização deste trabalho.

A Thomas Muller, do INMETRO, pela atenção e proveitosa discussão sobre questões relacionadas ao reconhecimento nacional e internacional de sistemas de metrologia e normalização.

Ao Mestre Pedro Paulo Almeida Silva, cuja dissertação de mestrado fundamentou a criação do Comitê Brasileiro de Normalização em Metrologia (ABNT/CB-53), pelo estímulo e inteligentes sugestões.

À direção do Instituto de Fomento e Coordenação Industrial, pelo estímulo e por ter-me liberado em regime de tempo parcial e por prover o endosso institucional para o desenvolvimento desta pesquisa de mestrado, que contribuiu para aprimoramento do sistema normativo do SISMETRA. Permanece a crença de que este trabalho de fato consolide-se como subsídio e embrião de um futuro sistema integrado de normalização para o Ministério da Defesa.

À Coordenação Central de Pós-Graduação e Pesquisa da Puc-Rio, pela bolsa de mestrado concedida no âmbito de convênio celebrado com o Centro Técnico Aeroespacial.

Às dedicadas Secretárias do PósMQI, Eliane Albernaz e Márcia Ribeiro, pelo carinho e eficiente apoio administrativo.

Ao Fundo Verde Amarelo, pelo apoio recebido por intermédio do projeto institucional concedido ao Programa de Pós-Graduação em Metrologia para Qualidade e Inovação da PUC-Rio, comprometido em alinhar suas pesquisas de mestrado com necessidades específicas que demandam conhecimentos avançados em metrologia, normalização e qualidade industrial.

Aos amigos Bianca Gonçalves, Índia Maria, Marcos José e Willians Portela pela amizade e companheirismo durante o curso. 


\section{Resumo}

Rangel, Elizete Gonçalves Lopes. Normalização em metrologia no Comando da Aeronáutica: diagnóstico e considerações sobre um modelo integrado. Rio de Janeiro, 2005. 196p. Dissertação de Mestrado Programa de Pós-Graduação em Metrologia. Área de concentração: Metrologia para Qualidade e Inovação. Pontifícia Universidade Católica do Rio de Janeiro (PUC-Rio).

Tendo como motivação a consolidação futura de um sistema integrado de metrologia para o Ministério da Defesa, a presente dissertação de mestrado diagnostica e propõe uma re-estruturação do sistema normativo do Sistema de Metrologia Aeroespacial (SISMETRA) pertencente ao Comando da Aeronáutica (COMAER). O sistema normativo em metrologia do SISMETRA, foco deste trabalho, tem como atribuição emitir normas técnicas (NTS) que padronizem a execução das atividades relacionadas com a metrologia no âmbito do COMAER. Fundamentado em ampla pesquisa bibliográfica e no diagnóstico do acervo normativo de Normas Técnicas do SISMETRA (NTS), foi possível mapear os principais sub-processos existentes no desenvolvimento de uma NTS e propor uma sistemática alternativa mais ágil e mais eficiente. Dois projetos de normas, elaborados segundo a nova filosofia proposta e com o objetivo de sistematizar os sub-processos existentes na criação de uma NTS, foram desenvolvidos como estudos de caso para comprovar a eficiência e eficácia do método proposto. $\mathrm{O}$ trabalho analisou, também, a conformidade do acervo normativo do SISMETRA a procedimentos internos e preceitos estabelecidos em normas nacionais e internacionais. Como fundamentos básicos do diagnóstico, a análise tomou como substrato os princípios internacionais da normalização e o "Standard Code", conforme caracterizado no Anexo 3 do Acordo de Barreiras Técnicas ao Comércio da Organização Mundial do Comércio. A análise investigou a aderência das NTS ao ABNT Guia 2 (Normalização e atividades relacionadas vocabulário geral) e a ABNT ISO/IEC Diretiva Parte 3 (Redação e apresentação de Normas Brasileiras) e a lógica processual relacionada ao desenvolvimento, adoção e aplicação de normas no âmbito do SISMETRA. No âmbito de uma pesquisa de demanda por normas em metrologia realizada junto aos quarenta e três (43) laboratórios de calibração que integram o SISMETRA em todo o território nacional, o trabalho identificou lacunas e deficiências no sistema vigente. Como resultado foram identificados 69 novos títulos de normas/procedimentos necessários para suprir vulnerabilidades e/ou atribuir maior racionalidade a sua operação. O desenvolvimento da pesquisa de 
demanda por normalização em metrologia, conduzida no âmbito do SISMETRA, de per se, já estimulou ampla reflexão sobre a relevância da atividade de normalização nos laboratórios do SISMETRA sediados em todo o território nacional. Este sistema é tomado como modelo para proposição de ações futuras visando a implementação de um sistema normativo integrado. Entendido como estratégico subsídio para planejamento futuro, acredita-se que o trabalho possa colaborar para o desenvolvimento de um sistema de metrologia integrado para o atual Ministério da Defesa.

\section{Palavras-chave}

Metrologia; Normalização; Sistema de metrologia aeroespacial (SISMETRA); Elaboração de normas; Pesquisa de Demanda por normas em metrologia no Comando da Aeronáutica. 


\section{Abstract}

Rangel, Elizete Gonçalves Lopes. Standardization in metrology for the the Brazilian Air Force: diagnosis and proposal of an integrated model. Rio de Janeiro, 2005. 196 pages. Master's Degree Dissertation Post - Graduate Programme in Metrology for Quality and Innovation. PUCRio (Rio de Janeiro Pontifical Catholic University of Rio de Janeiro).

Motivated by the future consolidation of an integrated metrology system for the Brazilian Defense Department, the present master's degree dissertation diagnoses and proposes the restructuring of SISMETRA's (Aerospace Metrology System) standardization system, which is part of the Brazilian Air Force (COMAER). SISMETRA's standardization system, focus of this master's degree research, issues technical standards to govern the activities related to metrology within the Brazilian Air Force's scope. Based on a wide bibliographic research and on the diagnosis of SISMETRA's technical standards (NTS) collection, it was possible to map the main processes underlaid in an NTS and proposed more agile and efficient procedure. Two sets of standards were prepared, according to the new proposed philosophy, as case study to prove the efficiency of the proposed method. This master's degree research also analyzed the conformity of SISMETRA's technical standards (NTS) in conformity with internal features and precepts established on national and international standards. As a basis to the diagnosis, the study took as substratum the international principles and the Standard Code, as characterized in Annex 3 of the Agreement on Technical Barriers to Trade, of the World Trade Organization (WTO). The study contemplated the adherence of SISMETRA's Technical Standards (NTS) to the Brazilian Standards (ABNT) Guide 2 and ABNT ISO/IEC Directive - Part 3 as well as the logic that lays underneath the development, the adoption and the application of norms within the scope of the Brazilian Air Force system's metrology. As a result of a survey on demand for metrology standards performed at the 43 calibrations laboratories which integrate SISMETRA nationwide, this master's degree research carried out a thorough review of the pros and cons of the existing system, and 69 titles of standards/procedures needed to reduce (or overcome) vulnerabilities and/or to improve its operation. The development of the undertaken survey on demand for standards in metrology, by itself, have stimulated a wide reflection and awareness on the importance of the standardization within SISMETRA's laboratories nationwide. This system is considered a model for future actions to erect an integrated standardization 
system. Seen as strategic subsidy, this master's degree research work may contribute to a future development of an integrated metrology system to meet the Ministry of Defense's needs.

\section{Key-Words}

Metrology; Standardization; Air-space metrology system (SISMETRA); Standards development; Survey on the demand for standardization in the Brazilian Air Force. 


\section{Sumário}

$\begin{array}{ll}\text { INTRODUÇÃO } & 23\end{array}$

Capítulo 1: Fundamentos da Metrologia e da Normalização 25

1.1. A metrologia 26

1.1.1. A metrologia: breve histórico 27

1.1.2. O Sistema Internacional de Unidades (SI) 29

1.1.3. Elementos da evolução do SI 31

1.1.4. A estrutura da metrologia científica 32

1.1.5. A infra-estrutura laboratorial brasileira 37

1.1.6. Conceitos e definições de interesse 40

$\begin{array}{lll}\text { 1.1.6.1. Calibração } & 40\end{array}$

1.1.6.2. Rastreabilidade 41

1.1.6.3. Incerteza de Medição 41

1.1.6.4. Padrão de Medição 42

1.1.6.5. Materiais de referência 43

1.2. A normalização 43

1.2.1. A normalização e sua estrutura mundial 44

1.2.2. A International Standardization for Organization (ISO) 47

1.2.3. O Processo de elaboração de normas da ISO 49

1.2.4. A International Electrotechnical Commission (IEC) 51

1.2.5. O processo de elaboração de normas da IEC 51

1.2.6. A International Telecomunication Union (ITU) 52

1.2.7. O processo de elaboração de normas da ITU 53

1.2.8. A normalização no cenário nacional 54

1.2.9. O processo de elaboração de normas da ABNT 57

1.3. As organizações internacionais de metrologia e normalização e avaliação da conformidade $\quad 59$

1.4 A prática internacional da normalização 62 
Capítulo 2: O Sistema de Metrologia do Comando da Aeronáutica

$2.1 \quad$ Breve Histórico do SISMETRA 69

2.2 A estrutura do SISMETRA: um sistema de metrologia 74

2.2.1. Os laboratórios do SISMETRA 79

2.2.2 SISMETRA: Instrumento de rastreabilidade ao SI 83

2.2.3 Sistema da Qualidade do SISMETRA 84

2.2.4 Confiabilidade Metrológica no SISMETRA 85

2.2.5 Gestão dos Padrões no SISMETRA 86

2.2.6 A rotina da atividade de calibração no SISMETRA 87

2.2.7 Manutenção da capacitação profissional 88

2.2.8 Reconhecimento nacional e internacional do SISMETRA 89

$\begin{array}{lll}2.3 & \text { O componente normalização no SISMETRA }\end{array}$

2.4 Análise crítica do SISMETRA na visão interna 91

2.4.1 Pré-diagnóstico do SISMETRA 92

2.4.2 Visão atual do Sistema e seus desafios 95

Capítulo 3: O Sistema Normativo em Metrologia do SISMETRA 97

$\begin{array}{lll}3.1 & \text { Os documentos de referência } & 98\end{array}$

3.2 O acervo normativo do SISMETRA 100

3.2.1 Aderência das NTS ao ABNT Guia 2:1998 106

3.2.2 Aderência das NTS à ABNT ISO/IEC Diretiva Parte 3:1995 109

3.3 O atual processo de criação de uma NTS 110

3.4 Proposição de nova sistemática para desenvolvimento de NTS 111

$\begin{array}{lll}3.5 & \text { Validação da nova sistemática proposta } & 114\end{array}$

Capítulo 4: Diagnóstico do Sistema Normativo do SISMETRA 117

4.1 Diagnóstico do acervo normativo do SISMETRA 117

$\begin{array}{lll}\text { 4.1.1 } & \text { Análise da conformidade à NTS 9-01 }\end{array}$

4.1.2 Análise da conformidade à NBR ISO/IEC 17025:2001 121

4.2 Síntese do diagnóstico do acervo normativo do SISMETRA 123 
Capítulo 5: Pesquisa de Demanda por NTS

$\begin{array}{lll}5.1 & \text { Fase \#1 da Pesquisa } & 126\end{array}$

$\begin{array}{lll}5.2 & \text { Fase \#2 da Pesquisa } & 127\end{array}$

$\begin{array}{lll}5.3 & \text { Fase \#3 da Pesquisa } & 129\end{array}$

$\begin{array}{lll}5.4 & \text { Fase \#4 da Pesquisa } & 130\end{array}$

$\begin{array}{lll}5.5 & \text { Síntese da Pesquisa de Demanda } & 134\end{array}$

Capítulo 6: Conclusões e Recomendações 137

$\begin{array}{ll}\text { REFERÊNCIAS BIBLIOGRÁFICAS } & 141\end{array}$

BIBLIOGRAFIA COMPLEMENTAR 145

$\begin{array}{ll}\text { APÊNDICES } & 147\end{array}$

$\begin{array}{lc}\text { ANEXOS } & 183\end{array}$ 


\section{Lista de Figuras}

Figura 1 - Unidades de base e unidades derivadas do SI.

Figura 2 - Estruturas formais de base da metrologia científica (Convenção do Metro).

Figura 3 - Sistema de Metrológico Mundial $\quad 36$

Figura 4 - Mapa da distribuição dos laboratórios de calibração da RBC. $\quad 38$

Figura 5 - Mapa da distribuição dos laboratórios de ensaio da RBLE. $\quad 39$

Figura 6 - Estrutura hierárquica dos padrões de medição. 42

Figura 7 - Níveis da normalização. $\quad 44$

Figura 8 - Organizações Regionais de Normalização. 46

Figura 9 - Estrutura do Sistema Brasileiro de Normalização. 55

Figura 10 - Processo e elaboração de normas técnicas nacionais. 58

Figura 11 - Organizações de Metrologia, Normalização e Avaliação da Conformidade.

Figura 12 - Estrutura orgânica do Centro Técnico Aeroespacial (CTA). $\quad 66$

Figura 13 - Estrutura organizacional do IFI. 66

Figura 14 - Estrutura Básica do Sistema de Metrologia Aeroespacial (SMA). 71

Figura 15 - SISMETRA: instrumento de confiabilidade aeroespacial. $\quad 75$

Figura 16 - A estrutura executiva do SISMETRA. 82

Figura 17 - Distribuição dos laboratórios do SISMETRA. 83

Figura 18 - Estrutura da confiabilidade metrológica e de rastreabilidade na ambiência do SISMETRA. 84

Figura 19 - Processo de desenvolvimento de uma NTS. 113

Figura 20 - Abrangência das fases da Pesquisa de Demanda por Normas em Metrologia. 


\section{Lista de Tabelas}

Tabela 1 - O ciclo de desenvolvimento de uma NTS: comparação entre o processo atual e o processo proposto.

Tabela 2 - NTS Aprovadas, segundo a categorização proposta.

Tabela 3 - Principais informações obtidas na Fase \#4 da Pesquisa de Demanda.

Tabela 4 - Resultado da Pesquisa de Demanda por Normas em Metrologia conduzida no âmbito do SISMETRA. 


\section{Lista de Quadros}

Quadro 1 - Unidades de base do SI. $\quad 30$

Quadro 2 - Unidades derivadas do SI. $\quad 31$

Quadro 3 - Laboratórios do SISMETRA. $\quad 81$

Quadro 4 - NTS aprovadas - Grupo "A" (NTS Gerenciais). 101

Quadro 5 - NTS aprovadas - Grupo "B” (Metrologia Básica). 102

Quadro 6 - NTS aprovadas - Grupo "C" (Metrologia Tempo de Freqüência). 102

Quadro 7 - NTS aprovadas - Grupo “D” (Metrologia Dimensional). 102

Quadro 8 - NTS aprovadas - Grupo "E" (Metrologia Elétrica). 104

Quadro 9 - NTS aprovadas - Grupo "F” (Metrologia Física). 105

Quadro 10 - NTS aprovadas - Grupo “G” (Metrologia Química). 105

Quadro 11 - Elementos de uma norma. 109

Quadro 12 - Acervo de NTS aprovadas a mais de cinco anos em out/2004. 119

Quadro 13 - Conformidade à NBR ISO/IEC 17025:2001. 122

Quadro 14 - Resultado obtido na Fase \#2 da pesquisa. 127

Quadro 15 - Resultado obtido na Fase \#3 da pesquisa. $\quad 129$

Quadro 16 - Resultado obtido na Fase \#4 da pesquisa. 131 


\title{
Lista de Abreviaturas e Símbolos
}

\author{
ABNT - Associação Brasileira de Normas Técnicas \\ ABNT/CB - Comitê Brasileiro de Normalização da ABNT \\ ABNT/ONS - Organismo de Normalização Setorial \\ ABENDE - Associação Brasileira de Ensaios Não Destrutivos \\ ACCSQ - Asian Consultative Committee for Standards and Quality (Comitê \\ Consultivo para Normas e Qualidade) \\ AFA - Academia da Força Aérea \\ AFNOR - Association Française de Normalisation (Associação Francesa de \\ Normalização) \\ AIAG - Automotive Industry Action Group (Grupo de Ação da Indústria \\ Automotiva) \\ AIDMO - Arab Industrial Development and Mining Organization \\ (Organização Árabe para o Desenvolvimento Industrial e a \\ Mineração) \\ AMN - Associação Mercosul de Normalização \\ ANATEL - Agência Nacional de Telecomunicações \\ ANSI - American National Standards Institute (Instituto Nacional de \\ Normalização dos EUA) \\ ANVISA - Agência Nacional de Vigilância Sanitária \\ ARSO - African Regional Organization for Standardization (Organização \\ Regional da África para a Normalização) \\ BAAN - Base Aérea de Anápolis (Goiás) \\ BABE - Base Aérea de Belém (Pará) \\ BABR - Base Aérea de Brasília (Distrito Federal) \\ BACG - Base Aérea de Campo Grande (Mato Grosso do Sul) \\ BACO - Base Aérea de Canoas (Rio Grande do Sul) \\ BAFZ - Base Aérea de Fortaleza (Ceará) \\ BAGL - Base Aérea do Galeão (Rio de Janeiro) \\ BAMN - Base Aérea de Manaus (Amazonas)
}


BANT - Base Aérea de Natal (Rio Grande do Norte)

BARF - Base Aérea de Recife (Pernambuco)

BASM - Base Aérea de Santa Maria (Rio Grande do Sul)

BASP - Base Aérea de São Paulo (São Paulo)

BAST - Base Aérea de Santos (São Paulo)

BASV - Base Aérea de Salvador (Bahia)

BIPM - Bureau International des Poids et Mesures (Bureau Internacional de Pesos e Medidas)

BSI - British Standards Institution (Instituto Britânico de Normalização)

CAC - Codex Alimentarius Comission (Comissão Codex Alimentarius)

CAvC - Divisão de Certificação de Aviação Civil do IFI

CB - Comitê Brasileiro

CBN - Comitê Brasileiro de Normalização

CE - Comissão de Estudo

CEET - Comissão de Estudo Especial Temporária

CEN - Europeam Committee for Standardization (Comitê Europeu para a Normalização)

CFA - Divisão de Coordenação de Fomento Aeroespacial do IFI

CGPM - Conference Generale dês Poids e Measures (Conferência Geral de Pesos e Medidas)

CGRE - Divisão de Acreditação de Laboratórios do INMETRO

CIEAR - Centro de Instrução e Adaptação da Aeronáutica

CIPM - Comité International des Poids et Mesures (Comitê Internacional de Pesos e Medidas)

CINDACTA I - Centro Integrado de Controle e Defesa do Espaço Aéreo I (Brasília)

CINDACTA II - Centro Integrado de Controle e Defesa do Espaço Aéreo II (Curitiba)

CINDACTA III - Centro Integrado de Controle e Defesa do Espaço Aéreo III (Recife)

CL - Subdivisão de Capacitação Laboratorial 
CLA - Centro de Lançamento de Alcântara

CLBI - Centro de Lançamento da Barreira do Inferno

CMA - Divisão de Confiabilidade Metrológica Aeroespacial

CNI - Confederação Nacional das Indústrias

CNN - Comitê Nacional de Normalização

COMAER - Comando da Aeronáutica

COMGAP - Comando Geral de Apoio

COMGAR - Comando Geral do Ar

COMGEP - Comando Geral do Pessoal

CONMETRO - Conselho Nacional de Metrologia, Normalização e Qualidade Industrial

COPANT - Comissão Pan-Americana de Normas Técnicas

CPA - Divisão de Produto Aeroespacial do IFI

CSG - Divisão de Sistemas de Gestão do IFI

CT - Comitê Técnico

CTA - Centro Técnico Aeroespacial

DECEA - Departamento de Controle do Espaço Aéreo

DEPED - Departamento de Pesquisas e Desenvolvimento

DEPENS - Departamento de Pesquisas e Ensino

DIN - Deutsch Institut für Normun (Instituto Alemão de Normalização)

DIRMAB - Diretoria de Material Aeronáutico e Bélico

DIS - Draft International Standard (Projeto de Norma Internacional)

EASC - Euro Asian Council for Standardization, Metrology and Certification (Conselho Euro-Asiático para Normalização, Metrologia e Certificação)

EEAR - Escola de Especialistas da Aeronáutica

ESCA - Engenharia de Sistemas de Controle e Automação S/C Ltda.

FDIS - Final Draft International Standard

GEIV - Grupo Especial de Inspeção de Vôo 
GT - Grupo de Trabalho

IAAC - International American Accreditation Cooperation (Cooperação Internacional Americana para a Acreditação)

IAE - Instituto de Aeronáutica e Espaço

IAF - International Accreditation Forum (Fórum Internacional de Acreditação)

IAOB - International Automotive Oversight Bureau (Bureau de Supervisão Internacional Automotiva)

IATCA - International Auditor and Training Certification Association (Associação Internacional de Auditores e Treinamento para Certificação)

IEAv - Instituto de Estudos Avançados

IEC - International Eletrotechnical Comission (Comissão Internacional de Eletrotécnica)

IFI - Instituto de Fomento e Coordenação Industrial (CTA)

ILAC - International Laboratory Accreditation Cooperation (Cooperação Internacional de Laboratórios de Acreditação)

IPQ - Instituto Português de Qualidade

INMETRO - Instituto Nacional de Metrologia, Normalização e Qualidade Industrial

IPEM - Instituto de Pesos e Medidas

ISO - International Organization for Standardization (Organização Internacional para Normalização)

ISOGUM - Guia para Expressão da Incerteza de Medição

ITA - Instituto Tecnológico de Aeronáutica

ITU - International Telecommunication Union (União Internacional de Telecomunicação)

IWA - International Workshop Agreement (Acordo Internacional obtido em Seminários)

LCC - Laboratório Central de Calibração

LNM - Laboratório Nacional de Metrologia

LRC - Laboratório Regional de Calibração

LSC - Laboratório Setorial de Calibração 
MCT - Ministério da Ciência e Tecnologia

MD - Ministério da Defesa

MNQ - Metrologia, Normalização e Qualidade Industrial

NBR ISO/IEC 17025:2001 - Requisitos gerais para competência de laboratórios de calibração e ensaio

NCSL - National Conference of Standards and Laboratories (Conferência Nacional de Padrões e Laboratórios, dos Estados Unidos)

NIST - National Institute for Standards and Technology (Instituto Nacional para Padrões e Tecnologia dos Estados Unidos)

NM - Seção de Normalização da Subdivisão de Capacitação Laboratorial

NTS - Norma Técnica do SISMETRA

OCC - Organismo de Certificação Credenciado

OCP - Organismos de Certificação de Produtos

OIML - Organisation Internationale de Métrologie Légale (Organização Internacional de Metrologia Legal)

OMC - Organização Mundial do Comércio

PASC - Pacific Área Standards Congress (Congresso de Normas da Área do Pacífico)

PAMA-AF - Parque Material Aeronáutico dos Afonsos (Rio de Janeiro)

PAMA-GL - Parque Material Aeronáutico do Galeão (Rio de Janeiro)

PAMA-LS - Parque Material Aeronáutico de Lagoa Santa (Minas Gerais)

PAMA-RF - Parque Material Aeronáutico de Recife (Pernambuco)

PAMA-SP - Parque Material Aeronáutico de São Paulo (São Paulo)

PAME - Parque Material Eletrônico da Aeronáutica (Rio de Janeiro)

PAS - Public Available Section (Seção Disponível ao público)

PBAC - Programa Brasileiro de Avaliação da Conformidade

PNS - Programa de Normalização Setorial

PÓS MQI - Programa de Pós-Graduação em Metrologia (Metrologia para a Qualidade e Inovação) da PUC-Rio

PTB - Physikalisch-Technische Bundesanstalt (Instituto Nacional de Metrologia da Alemanha) 
PUC-Rio - Pontifícia Universidade Católica do Rio de Janeiro

RBC - Rede Brasileira de Calibração

RBLE - Rede Brasileira de Laboratórios de Ensaio

SBAC - Sistema Brasileiro de Avaliação da Conformidade

SBN - Sistema Brasileiro de Normalização

SEMETRA - Seminário de Metrologia Aeroespacial

SI - Sistema Internacional de Unidades

SIM - Sistema Interamericano de Metrologia

SINMETRO - Sistema Nacional de Metrologia, Normalização e Qualidade Industrial

SRPV-MN - Serviço Regional de Proteção ao Vôo de Manaus (Amazonas)

SRPV-RJ - Serviço Regional de Proteção ao Vôo do Rio de Janeiro (Rio de Janeiro)

SRPV-SP - Serviço Regional de Proteção ao Vôo de São Paulo (São Paulo)

SISCEAB - Sistema de Controle do Espaço Aéreo Brasileiro

SISMEFA - Sistema de Metrologia das Forças Armadas

SISMETRA - Sistema de Metrologia Aeroespacial

SMA - Sistema de Metrologia Aeroespacial

TBT - Agreement on Technical Barriers to Trade (Acordo sobre Barreiras Técnicas no Comércio, da Organização Mundial de Comércio)

TIB - Tecnologia Industrial Básica

TR - Technical Report (Relatório Técnico)

TS - Technical Specification (Especificação Técnica)

VIM - Vocabulário Internacional de Termos Fundamentais e Gerais de Metrologia

WHO - Word Health Organization (Organização Mundial da Saúde)

WTO - World Trade Organization (Organização Mundial do Comércio) 